\title{
CIRURGIA CARDÍACA: PERFIL CLÍNICO DOS PACIENTES E ACOMPANHAMENTO EM 30 DIAS
}

\author{
Cardiac surgery: clinical profile of patients and 30-day follow-up \\ Cirugía cardíaca: perfil clinico del paciente y seguimiento en el período de 30 días \\ Sônia Regina Barcellos ${ }^{*} \oplus$, Angelita Paganin Costanzi ${ }^{\oplus}$, Francieli Alessandra Strelow ${ }^{3} \odot$,

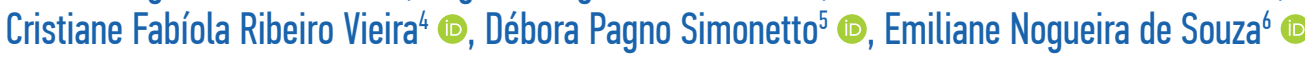

RESUMO: Objetivo: Caracterizar o perfil clínico dos pacientes submetidos à cirurgia cardíaca no perioperatório e descrever o acompanhamento após 30 dias da alta hospitalar. Método: Estudo retrospectivo, com amostra de 54 pacientes, de ambos os sexos, com idade $\geq 18$ anos, submetidos à cirurgia cardíaca. As variáveis analisadas foram: sociodemográficas, clínicas e relacionadas ao perioperatório e seguimento em 30 dias. Resultados: A média de idade dos pacientes foi de 65,5 \pm 15 anos; a maioria do sexo masculino (79,6\%). Fatores de risco mais prevalentes: hipertensão (72,2\%), dislipidemia (48,1\%) e cardiopatia isquêmica (31,5\%). Os procedimentos valvares tiveram maior prevalência (50,0\%). A complicação mais prevalente no pós-operatório foi arritmia (18,5\%). No pós-alta, o uso das estatinas predominou (78,4\%), seguido de antiagregante plaquetário (50,9\%) e anticoagulante (31,3\%). Após 30 dias da alta hospitalar, houve $11,7 \%$ de readmissões, sendo a infecção respiratória/derrame pleural e a infecção de sítio cirúrgico as principais causas de reinternação. Conclusão: Os pacientes cardícos foram na maioria homens, idosos e que apresentavam comorbidades; arritmia foi a principal complicação após a cirúrgia. A incidência de readmissão após 30 dias da alta foi relacionada a comprometimentos pulmonares e infecciosos.

Palavras-chave: Cuidados de enfermagem. Enfermagem perioperatória. Procedimentos cirúrgicos cardíacos.

ABSTRACT: Objective: To characterize the clinical profile of patients who underwent cardiac surgery in the perioperative period and describe the follow-up after 30 days of hospital discharge. Method: Retrospective study, with a sample of 54 patients, both male and female, aged $\geq 18$ years. The analyzed variables were: sociodemographic, clinical and those related to the perioperative period and 30-day follow-up. Results: The mean age of patients was $65.5 \pm 15$ years; most were male $(79.6 \%)$. The most prevalent risk factors were: hypertension $(72.2 \%)$, dyslipidemia $(48.1 \%)$ and ischemic heart disease (31.5\%). Valve procedures had higher prevalence $(50.0 \%)$. The most prevalent complication in the postoperative period was arrhythmia (18.5\%). At post-discharge, statins (78.4\%) were the most prevalent medication, followed by antiplatelet agents (50.9\%) and anticoagulants (31.3\%). After 30 days of hospital discharge, the percentage of readmission was $11.7 \%$, being the main causes of readmission respiratory infection/pleural effusion and surgical site infection. Conclusion: Surgical patients were mostly elderly men who had comorbidities; arrhythmia was the main complication found after surgery. The incidence of readmission 30 days after discharge was related to pulmonary complications and infections.

Keywords: Nursing care. Perioperative nursing. Cardiac surgical procedures.

RESUMEN: Objetivo: Caracterizar el perfil clínico de los pacientes sometidos a cirugía cardíaca en el período perioperatorio y describir el seguimiento a los 30 días del alta hospitalaria. Método: Estudio retrospectivo, con una muestra de 54 pacientes, de ambos sexos, $\geq 18$ años, sometidos a cirugía cardíaca. Las variables analizadas fueron: sociodemográficas, clínicas y relacionadas con el período perioperatorio y seguimiento en 30 días. Resultados: La edad

\footnotetext{
'Mestranda do Programa de Pós-Graduação em Enfermagem da Universidade Federal de Ciências da Saúde de Porto Alegre (UFCSPA). Monitora de enfermagem do Centro Cirúrgico e Sala de Recuperação do Hospital Unimed - Caxias do Sul (RS), Brasil.

${ }^{2}$ Doutora pelo Programa de Pós-Graduação de Enfermagem da Universidade Federal do Rio Grande do Sul (UFRGS). Coordenadora dos Centros Cirúrgicos, Hemodinâmica e Endoscopia do Hospital Unimed - Caxias do Sul (RS), Brasil.

${ }^{3}$ Especialista em Terapia Intensiva pela Universidade de Caxias do Sul. Enfermeira assistencial no centro cirúrgico do Hospital Unimed - Caxias do Sul (RS), Brasil. ¿Enfermeira pelo Centro Universitário da Serra Gaúcha. Coordenadora do Centro Cirúrgico e Sala de Recuperação do Hospital Unimed - Caxias do Sul (RS), Brasil.

${ }^{5}$ Especialista em Terapia Intensiva pelo Hospital Moinhos de Vento. Enfermeira assistencial da Sala de Recuperação do Hospital Unimed - Caxias do Sul (RS), Brasil.

${ }^{6}$ Doutora em Ciências da Saúde: cardiologia pela UFRGS. Docente do Programa de Pós-Graduação em Enfermagem da UFCSPA - Porto Alegre (RS), Brasil.

*Autora correspondente: soniabarcellos8@gmail.com

Recebido: 13/07/2020 - Aprovado: 26/10/2020

https://doi.org/10.5327/Z1414-4425202100010007
} 
media de los pacientes fue de 65,5 \pm 15 años; la mayoría eran hombres (79,6\%). Factores de riesgo más prevalentes: hipertensión (72,2\%), dislipidemia $(48,1 \%)$ y cardiopatía isquémica (31,5\%). Los procedimientos valvulares tuvieron una mayor prevalencia (50,0\%). La complicación más prevalente en el postoperatorio fue la arritmia (18,5\%). Al alta, predominó el uso de estatinas $(78,4 \%)$, seguido de antiagregantes plaquetarios (50,9\%) y anticoagulantes (31,3\%). Treinta días después del alta hospitalaria hubo un 11,7\% de reingresos, siendo la infección respiratoria / derrame pleural y la infección del sitio quirúrgico las principales causas de reingreso. Conclusión: Los pacientes cardíacos eran en su mayoría hombres, ancianos y con comorbilidades; la arritmia fue la principal complicación después de la cirugía. La incidencia de reingreso a los 30 días del alta se relacionó con alteraciones pulmonares e infecciosas. Palabras Clave: Atención de enfermería. Enfermería perioperatoria. Procedimientos quirúrgicos cardíacos.

\section{INTRODUÇÃo}

As doenças crônicas englobam um conjunto de patologias, entre as quais, as doenças cardiovasculares, que possuem elevadas taxas de morbidade e mortalidade. A Organização Mundial da Saúde (OMS) aponta as doenças cardiovasculares como as principais causas de morte no mundo. O levantamento mais recente, com dados de 2015, mostra que 17,7 milhões de óbitos ocorreram em decorrência de doenças cardiovasculares ${ }^{1}$. No Brasil, em 2019, mais de 289 mil pessoas morreram em virtude das doenças cardiovasculares e suas complicações. Tais doenças apresentam múltiplas etiologias, associadas a incapacidades funcionais que, consequentemente, potencializam impactos socioeconômicos, culturais e ambientais.

As cardiopatias representam um grande problema de saúde pública, sendo necessário o uso de métodos eficazes, com a finalidade de potencializar ações resolutivas, acerca da assistência à saúde. As estimativas apontam para a possibilidade de que, nos próximos 20 anos, no Brasil, o número de idosos ultrapasse os 30 milhões de pessoas, devendo representar quase $13 \%$ da população. Considerando que a expectativa de vida vem aumentando, consequentemente, os idosos necessitam de algum tipo de intervenção cardiovascular, acarretando maior longevidade e melhora da qualidade de vida ${ }^{2,3}$.

Estudos mostram mudanças no perfil clínico dos pacientes. Com novas tecnologias e incrementos dos processos assistenciais, a cirurgia cardíaca tem sido indicada mais tardiamente, o que faz com que os pacientes tenham inúmeras patologias associadas. No entanto, o maior número de comorbidades influencia os desfechos clínicos, havendo maior número de complicações no pós-operatório ${ }^{4}$.

O tratamento cirúrgico busca aumentar a sobrevida, com melhora na qualidade de vida dos pacientes. Entre as cirurgias cardíacas, a cirurgia de revascularização do miocárdio possui maior prevalência ( $64 \%$ no Brasil), seguida pelas correções valvares. Considerando as limitações geradas pelo procedimento cirúrgico, é necessário que o paciente tenha sua independência funcional garantida, permitindo o retorno às atividades da vida diária ${ }^{5-7}$.

Nesse sentido, dados extraídos do DATASUS, nos últimos cinco anos (entre 2014 e 2019), em uma análise populacional brasileira, evidenciam que foram realizados 72.157 procedimentos cirúrgicos cardíacos, sendo $43,57 \%$ na Região Sudeste do país. No último ano (2019), houve 32.732 casos cirúrgicos, com 7,05\% de taxa de mortalidade. As doenças cardiovasculares são responsáveis por inúmeras readmissões e elevado tempo de permanência hospitalar ${ }^{7}$.

A maioria dos pacientes submetidos à cirurgia cardíaca apresenta nível elevado de ansiedade e expectativa em relação ao procedimento, o que pode comprometer sua evolução. Dessa maneira, a equipe de enfermagem é responsável por transmitir informações aos pacientes, ampliando o conhecimento e tranquilizando-os nos períodos pré, trans e pós-operatório ${ }^{8}$. Para tanto, os processos assistenciais necessitam de aprimoramento contínuo, sendo requerido o acompanhamento desses pacientes tanto no perioperatório quanto após a alta hospitalar. Os primeiros 30 dias do pós-alta são importantes para a readaptação do paciente à vida cotidiana, pela complexidade do procedimento anestésico-cirúrgico e pela internação hospitalar à qual foi submetido.

A enfermagem perioperatória desempenha papel relevante nesse cenário, não somente pela prestação direta de assistência aos pacientes submetidos às cirurgias cardíacas, como também fornecendo orientações e educação a esses e aos seus familiares durante a internação e após a alta hospitalar.

\section{OBJETIVO}

Caracterizar o perfil clínico dos pacientes submetidos à cirurgia cardíaca no período perioperatório e descrever o acompanhamento a esses pacientes após 30 dias da alta hospitalar. 


\section{MÉTODO}

Estudo retrospectivo, realizado em um hospital geral, de grande porte, situado na Região Sul do Brasil, cidade de Caxias do Sul, estado do Rio Grande do Sul (RS). A instituição é referência em cirurgia cardíaca desde 2004, dispondo de uma unidade de dor torácica com seis leitos e duas unidades de terapia intensiva adulto, com 10 leitos cada, onde se recuperam os pacientes submetidos à cirurgia cardíaca. A demanda cirúrgica é aproximadamente de seis a oito cirurgias ao mês. O período analisado foi de março a setembro de 2019 .

Incluíram-se pacientes de ambos os sexos, com idade $\geq 18$ anos, submetidos a procedimentos de cirurgia cardíaca (cirurgia de revascularização do miocárdio, cirurgias valvares, aneurismas e cirurgias combinadas), de forma eletiva ou urgência. Excluíram-se pacientes com cirurgias relacionadas a cardiopatias congênitas.

A amostra foi composta pela totalidade de 54 pacientes que atenderam aos critérios de inclusão no período. Realizou-se a coleta com base em dados secundários construída e atualizada pela equipe de enfermagem do centro cirúrgico (CC), que acompanha os pacientes por até um ano após o procedimento anestésico-cirúrgico. O segmento de pacientes prevê um contato telefônico realizado pela enfermeira em 1, 3, 6 e 12 meses após a realização da cirurgia. Para este estudo, considerou-se apenas o seguimento dos primeiros 30 dias.

As variáveis analisadas no pré-operatório foram as sociodemográficas e as clínicas; as variáveis no transoperatório foram o tempo de circulação extracorpórea (CEC) e o tempo de clampleamento; as variáveis no pós-operatório foram o tempo de extubação após a admissão na unidade de terapia intensiva (UTI), a utilização de fármacos, o uso de dispositivo de assistência circulatória, o uso de hemoderivados, as complicações (cardiocirculatórias, renais, respiratórias e relacionadas ao procedimento) e a ocorrência de óbito. No seguimento de pacientes em 30 dias, avaliaram-se as readmissões hospitalares, os eventos cardiopulmonares e a ocorrência de infecção de sítio cirúrgico (ISC).

Os dados são apresentados por meio de números absolutos (n) e relativos (\%), média e desvio padrão, quando normalmente distribuídos, ou mediana com percentis $25 \mathrm{e}$ 75, quando não há distribuição normal. Verificou-se a normalidade pelo teste Shapiro-Wilk. Aferiram-se os dados por meio do software Statistical Package for Social Sciences (SPSS), versão 25.
O presente estudo caracteriza-se como uma subanálise de um projeto maior, com a mesma amostra, o qual seguiu as recomendações da Resolução $n^{\circ}$ 466/2012, do Conselho Nacional da Saúde, tendo sido aprovada pelo Comitê de Ética em Pesquisa da instituição sede da pesquisa, via Plataforma Brasil - Certificado de Apresentação para Apreciação Ética (CAAE) 33329114.3.0000.5523 / Parecer 3.935.050.

\section{RESULTADOS}

Analisaram-se os dados de 54 pacientes, e o tempo médio de permanência na UTI foi de $4,19 \pm 4,31$ dias, e o total de inter-

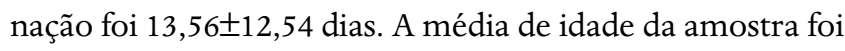
de $65,5 \pm 15$ anos. O sexo predominante foi o masculino, com $43(79,6 \%)$ pacientes. O procedimento de maior prevalência foi a cirurgia valvar, com $27(50,0 \%)$ pacientes, seguida pela cirurgia de revascularização do miocárdio (CRM), com 17 $(31,5 \%)$ pacientes. A maioria das cirurgias foi eletiva -50 $(92,5 \%)$. As caracterizações sociodemográfica e clínica da amostra estão descritas na Tabela 1.

Em relação às variáveis intraoperatórias, o tempo médio de CEC foi de 109 \pm 39 minutos, e o tempo de clampeamento

Tabela 1. Caracterização sociodemográfica e clínica no pré-operatório de pacientes submetidos à cirurgia cardíaca $(n=54)$.

\begin{tabular}{|l|c|c|c|c|}
\hline Variáveis & $\begin{array}{c}\text { Total } \\
\mathbf{n = 5 4} \\
\mathbf{n}(\%)\end{array}$ & $\begin{array}{c}\text { CRM } \\
\mathbf{n = 1 7} \\
\mathbf{n}(\%)\end{array}$ & $\begin{array}{c}\text { Valvar } \\
\mathbf{n = 2 7} \\
\mathbf{n}(\%)\end{array}$ & $\begin{array}{c}\text { Outras } \\
\mathbf{n = 1 0} \\
\mathbf{n}(\%)\end{array}$ \\
\hline Procedimentos & $\begin{array}{c}54 \\
(100,0)\end{array}$ & $17(31,5)$ & $27(50,0)$ & $10(18,5)$ \\
\hline $\begin{array}{l}\text { Sexo } \\
\text { masculino }\end{array}$ & $43(79,6)$ & $15(88,2)$ & $18(66,7)$ & $10(100,0)$ \\
\hline Idade* & $65,5 \pm 15$ & $65 \pm 13$ & $63 \pm 14$ & $56 \pm 16$ \\
\hline HAS & $39(72,2)$ & $14(82,4)$ & $21(77,8)$ & $4(40,0)$ \\
\hline Dislipidemia & $26(48,1)$ & $12(70,6)$ & $11(40,7)$ & $3(30,0)$ \\
\hline $\begin{array}{l}\text { Cardiopatia } \\
\text { isquêmica }\end{array}$ & $17(31,5)$ & $17(100,0)$ & $0(0)$ & $0(0)$ \\
\hline Tabagismo & $15(27,8)$ & $6(35,3)$ & $5(18,5)$ & $4(40,0)$ \\
\hline DM & $14(25,9)$ & $9(52,9)$ & $4(14,8)$ & $1(10,0)$ \\
\hline Etilismo & $4(7,4)$ & $2(11,8)$ & 0 & $2(20,0)$ \\
\hline IRC & $1(1,9)$ & $0(0)$ & $1(3,7)$ & 0 \\
\hline DPOC & $1(1,9)$ & $0(0)$ & $0(0)$ & $1(10,0)$ \\
\hline $\begin{array}{l}\text { Fração de } \\
\text { ejęãa }\end{array}$ & $60 \pm 1$ & $60 \pm 1$ & $63 \pm 1$ & $63 \pm 0$ \\
\hline
\end{tabular}

*média e desvio padrão; CRM: cirurgia de revascularização do miocárdio; HAS: hipertensão arterial sistêmica; DM: diabetes mellitus; IRC: insuficiência renal crônica; DPOC: doença pulmonar obstrutiva crônica. 
foi, em média, de $76 \pm 28$ minutos. O maior tempo de CEC foi nos pacientes submetidos à CRM (105 $\pm 44 \mathrm{~min})$, seguidos pelos pacientes submetidos a cirurgias valvares $(100 \pm 33 \mathrm{~min})$. Em relação à extubação, evidenciamos que 37 (68,5\%) pacientes foram extubados nas seis primeiras horas de pós-operatório, sendo as cirurgias valvares com maior número de extubações - 21 (77,8\%) -, conforme preconizado.

As medicações mais utilizadas foram vasopressoras, majoritariamente nos pacientes submetidos à cirurgia valvar $(21 / 77,8 \%)$, seguidas pelas medicações vasodilatadoras ( 9 / 52,9\%), em cirurgias de CRM. No que diz respeito à transfusão sanguínea, identificamos que $11(20,4 \%)$ pacientes necessitaram dessa terapia. A grande maioria dos pacientes teva alta da UTI com pontuação satisfatória no Swift (Tabela 2).

A mortalidade no pós-operatório imediato (POI) foi de três $(5,5 \%)$ pacientes. Em relação às complicações pós-operatórias em até 48 horas, destaca-se a arritmia (10 / 18,5\%), seguida de sangramento e vasoplegia (6/11,1\%). Tais dados estão na Tabela 3.

Tabela 2. Dados referentes à assistência pós-operatória imediata na unidade de terapia intensiva (UTI) $(n=54)$.

\begin{tabular}{|c|c|c|c|c|}
\hline Variáveis & $\begin{array}{l}\text { Total } \\
n=54 \\
n(\%)\end{array}$ & $\begin{array}{l}\text { CRM } \\
n=17 \\
n(\%)\end{array}$ & $\begin{array}{c}\text { Valvar } \\
n=27 \\
n(\%)\end{array}$ & $\begin{array}{c}\text { Outras } \\
n=10 \\
n(\%)\end{array}$ \\
\hline $\begin{array}{l}\text { Extubação em } \\
\text { até } 6 \text { horas }\end{array}$ & $37(68,5)$ & $8(47,1)$ & $21(77,8)$ & $8(80,0)$ \\
\hline Vasopressor & $47(87,0)$ & $16(94,1)$ & $21(77,8)$ & $10(100,0)$ \\
\hline Vasodilatador & $20(37,0)$ & $9(52,9)$ & $8(29,6)$ & $3(30,0)$ \\
\hline Inotrópico & $8(14,8)$ & $5(29,4)$ & $3(11,1)$ & $0(0)$ \\
\hline Precedex & $10(18,5)$ & $5(29,4)$ & $3(11,1)$ & $2(20,0)$ \\
\hline Fentanil & $5(9,3)$ & $2(11,8)$ & $3(11,1)$ & $0(0)$ \\
\hline Midazolan & $3(5,6)$ & $3(17,6)$ & $0(0)$ & $0(0)$ \\
\hline $\begin{array}{l}\text { Balão } \\
\text { intraórtico }\end{array}$ & $1(1,9)$ & $1(5,9)$ & $0(0)$ & $0(0)$ \\
\hline $\begin{array}{l}\text { Terapia de } \\
\text { substituição } \\
\text { renal }\end{array}$ & $1(1,9)$ & $0(0)$ & $1(3,7)$ & $0(0)$ \\
\hline $\begin{array}{l}\text { Transfusão } \\
\text { sanguínea }\end{array}$ & $11(20,4)$ & $5(29,4)$ & $4(14,8)$ & $2(20,0)$ \\
\hline $\begin{array}{l}\text { Swift de } \\
\text { alta da UTI }\end{array}$ & -- & -- & -- & -- \\
\hline Baixo & $46(85,2)$ & $14(82,4)$ & $24(88,9)$ & $8(80,0)$ \\
\hline Alto $(\geq 15)$ & $8(14,8)$ & $3(17,6)$ & $3(11,1)$ & $2(20,0)$ \\
\hline
\end{tabular}

No que tange às complicações apresentadas pelos pacientes após 48 horas até a alta hospitalar, predominou a arritmia, que ocoreu em sete (13,7\%) pacientes (Tabela 4).

Entre as medicações que os pacientes da amostra fazem uso no domicílio, após a alta hospitalar do evento cirúrgico, destacam-se as estatinas ( $40 / 78,4 \%$ ), os antiagregantes plaquetários (26/50\%), os inibidores da enzima conversora em angiotensina ( $20 / 39,2 \%)$ e os anticoagulantes orais $(16 / 31,3 \%)$.

Seis $(11,7 \%)$ pacientes foram readmitidos 30 dias após alta hospitalar por complicações. As principais complicações nesse período foram: infecção respiratória/derrame pleural (3 / 5,9\%) e ISC (3 / 5,9\%). Demais dados constam na Tabela 5.

Tabela 3. Complicações apresentadas pelos pacientes no pós-operatório (até 48 horas) $(n=54)$.

\begin{tabular}{|l|c|c|c|c|} 
Variáveis & $\begin{array}{c}\text { Total } \\
\mathbf{n = 5 4} \\
\mathbf{n}(\%)\end{array}$ & $\begin{array}{c}\text { CRM } \\
\mathbf{n = 1 7} \\
\mathbf{n}(\%)\end{array}$ & $\begin{array}{c}\text { Valvar } \\
\mathbf{n = 2 7} \\
\mathbf{n}(\%)\end{array}$ & $\begin{array}{c}\text { Outras } \\
\mathbf{n = 1 0} \\
\mathbf{n}(\%)\end{array}$ \\
\hline Arritmia & $10(18,5)$ & $2(11,8)$ & $7(25,9)$ & $1(10,0)$ \\
\hline $\begin{array}{l}\text { Sangramento } \\
\text { Vasoplegia }\end{array}$ & $6(11,1)$ & $2(11,8)$ & $3(11,1)$ & $1(10,0)$ \\
\hline $\begin{array}{l}\text { nsuficiência } \\
\text { renal }\end{array}$ & $3(5,6)$ & $1(5,9)$ & $2(7,4)$ & $0(0)$ \\
\hline $\begin{array}{l}\text { Insuficiência } \\
\text { respiratória } \\
\text { aguda }\end{array}$ & $3(5,5)$ & $1(5,9)$ & $1(3,7)$ & $1(10,0)$ \\
\hline \begin{tabular}{l} 
Óbito \\
\hline
\end{tabular} & $3(5,5)$ & $1(5,8)$ & $1(3,7)$ & $1(10,0)$ \\
\hline
\end{tabular}

CRM: cirurgia de revascularização do miocárdio.

Tabela 4. Complicações apresentadas pelos pacientes após 48 horas até a alta hospitalar $(n=51)$.

\begin{tabular}{|l|c|c|c|c|}
\hline Variáveis & $\begin{array}{l}\text { Total } \\
\mathbf{n = 5 1} \\
\mathbf{n}(\%)\end{array}$ & $\begin{array}{c}\text { CRM } \\
\mathbf{n = 1 6} \\
\mathbf{n}(\%)\end{array}$ & $\begin{array}{c}\text { Valvar } \\
\mathbf{n = 2 6} \\
\mathbf{n}(\%)\end{array}$ & $\begin{array}{c}\text { Outras } \\
\mathbf{n = 1 0} \\
\mathbf{n}(\%)\end{array}$ \\
\hline Arritmia & $7(13,7)$ & $2(12,5)$ & $4(15,4)$ & $1(11,1)$ \\
\hline $\begin{array}{l}\text { Insuficiência } \\
\text { renal }\end{array}$ & $3(5,9)$ & $1(6,3)$ & $1(3,8)$ & $1(11,1)$ \\
\hline $\begin{array}{l}\text { Insuficiência } \\
\text { respiratória }\end{array}$ & $1(2,0)$ & $1(6,3)$ & $0(0)$ & $0(0)$ \\
\hline $\begin{array}{l}\text { Infecção de } \\
\text { ferida operatória }\end{array}$ & $1(2,0)$ & $0(0)$ & $0(0)$ & $1(11,1)$ \\
\hline $\begin{array}{l}\text { Edema agudo } \\
\text { de pulmão }\end{array}$ & $2(3,9)$ & $2(12,5)$ & $0(0)$ & $0(0)$ \\
\hline Pneumonia & $1(1,9)$ & $0(0)$ & $1(3,8)$ & $0(0)$ \\
\hline
\end{tabular}

CRM: cirurgia de revascularização do miocárdio. 
Tabela 5. Readmissões e complicações dos pacientes em 30 dias após a alta hospitalar $(n=51)$.

\begin{tabular}{|l|c|c|c|c|}
\hline Variáveis & $\begin{array}{c}\text { Total } \\
\mathbf{n = 5 1} \\
\mathbf{n}(\%)\end{array}$ & $\begin{array}{c}\text { CRM } \\
\mathbf{n = 1 6} \\
\mathbf{n}(\%)\end{array}$ & $\begin{array}{c}\text { Valvar } \\
\mathbf{n = 2 6} \\
\mathbf{n}(\%)\end{array}$ & $\begin{array}{c}\text { Outras } \\
\mathbf{n = 9} \\
\mathbf{n}(\%)\end{array}$ \\
\hline $\begin{array}{l}\text { Readmissão } \\
\text { hospitalar } \\
\text { em 30 dias }\end{array}$ & $6(11,7)$ & $1(6,3)$ & $2(7,7)$ & $2(22,2)$ \\
\hline $\begin{array}{l}\text { Derrame pleural } \\
\text { e infecção } \\
\text { respiratória }\end{array}$ & $3(5,9)$ & $1(6,3)$ & $2(7,7)$ & $0(0)$ \\
\hline $\begin{array}{l}\text { Infecção de } \\
\text { ferida operatória }\end{array}$ & $3(5,9)$ & $1(6,3)$ & $0(0)$ & $2(22,2)$ \\
\hline $\begin{array}{l}\text { CRM: cirurgia de revascularização do miocárdio. } \\
\end{array}$
\end{tabular}

\section{DISCUSSÃO}

A pesquisa com dados de 54 pacientes submetidos a cirurgias cardíacas demonstrou predomínio do sexo masculino, com idade média de 65 anos. A comorbidade mais prevalente foi a hipertensão arterial sistêmica (HAS), seguida de dislipidemia. Entre as cirurgias cardíacas mais prevalentes, destacaram-se as trocas valvares e a CRM. Outros estudos, também realizados com pacientes submetidos à cirurgia cardíaca, mostraram que há predomínio de homens idosos, porém o procedimento de maior incidência foi a CRM, sendo a HAS, o diabetes mellitus (DM) e a dislipidemia as comorbidades mais prevalentes ${ }^{9-11}$.

Em relação ao transoperatório, observa-se que os tempos de CEC e clampeamento assemelham-se à média encontrada na literatura. Em um estudo do tipo caso-controle, que comparou pacientes não pertencentes ao protocolo com pacientes que entraram para o protocolo Enhanced Recovery After Surgery (ERAS), os autores evidenciaram elevado tempo de CEC e de clampeamento, semelhante ao estudo vigente. O protocolo ERAS tem a finalidade de melhorar desfechos e complicações no pós-operatório, propondo cuidados específicos no pré-operatório ${ }^{12}$. No entanto, outro estudo, realizado no noroeste do estado do RS, evidenciou que os tempos de CEC foram menores, tendo em vista o perfil dos pacientes atríbuído à CRM. O elevado tempo de CEC está associado à doença inflamatória sistêmica, podendo causar complicações, como vasoplegia, doença renal, entre outras, e aumentando o tempo de internação ${ }^{13}$.

Quanto ao tempo de extubação, estudos apontam para um intervalo superior ao que apresentamos neste estudo. Estudo realizado na Região Sudoeste do Brasil, que avaliou
200 pacientes em pós-operatório de cirurgia cardíaca, mostrou tempo médio de extubação superior a 12 horas ${ }^{10}$. Já outro estudo, que avaliou 47 pacientes submetidos à cirurgia cardíaca valvar, evidenciou tempo médio de 14,5 horas. A extubação precoce ou menor do que 6 horas favorece desfechos, como redução do tempo de internação, enquanto a extubação realizada após 6 horas está associada a complicações no pós-operatório 3,10,14.

Com relação às medicações utilizadas no POI, os vasopressores assumiram lugar de destaque, seguidos pelos vasodilatadores (nitroglicerina). Nas CRM com a inserção da artéria mamária como ponte, é necessário o uso de vasodilatadores para manter a permeabilidade das pontes. Já as medicações vasopressoras são necessárias no POI dada a instabilidade hemodinâmica. O vasopressor mais utilizado no POI é a noradrenalina, já o vasodilatador mais utilizado é a nitroglicerina, ambos por via endovenosa em acesso central ${ }^{10}$.

Quanto à necessidade de hemoderivados no POI, a metade dos pacientes deste estudo necessitou de transfusão sanguínea, majoritariamente aqueles submetidos à CRM. O sangramento no pós-operatório é o principal motivo pelo qual os pacientes recebem hemocomponentes ${ }^{15}$. A transfusão de hemocomponentes está associada à reação transfusional, à infecção pós-operatória, ao aumento da morbimortalidade pós-operatória, ao risco de imunossupressão e ao maior tempo de internação ${ }^{15}$.

A fibrilação atrial (FA) é uma das complicações mais comuns no POI, podendo estar presente também no pós-operatório tardio. Conforme as II Diretrizes Brasileiras de Fibrilação Atrial, no período pós-operatório, a FA pode ocorrer entre 24 e 72 horas após a cirurgia, principalmente em cirurgias valvares ${ }^{16}$.

Corroborando os dados desta pesquisa, outro estudo que aborda pacientes no pós-operatório identificou que a complicação mais frequente nos pacientes submetidos a cirurgias cardíacas foram as arritmias, destacando-se a FA, presente em $45 \%$ deles $^{17}$. Além das arritmias, outras complicações podem ser encontradas no pós-operatório, como o sangramento e a síndrome vasoplégica; ambas assumem lugar de destaque pela elevada taxa de mortalidade. Um estudo que descreve desfechos clínicos de pacientes em uma instituição no noroeste do RS configurou o sangramento como a segunda complicação mais frequente no pós-operatório, podendo imergir de inúmeras causas, desde técnicas cirúrgicas, distúrbios de coagulação, uso excessivo de heparina e complicações oriundas do tempo elevado de CEC. Essas complicações podem estar relacionadas a doenças preexistentes, tendo em vista que muitos pacientes 
possuem múltiplas comorbidades. Sendo assim, é importante que os pacientes estejam compensados clinicamente ${ }^{13}$.

Um estudo que objetivou descrever as complicações no POI de cirurgias cardíacas, com uma população predominantemente masculina e submetida à CRM, demonstrou que os pacientes apresentaram distúrbios hidroeletrolíticos, seguidos de arritmias cardíacas, como as principais complicações ${ }^{18}$. Já outro estudo objetivou descrever complicações em 2.648 pacientes que realizaram cirurgia cardíaca em uma instituição no estado de São Paulo, descrevendo como principais complicações a lesão renal aguda e as patologias pulmonares oriundas de infecção respiratória e derrame pleural, ambas relacionadas ao elevado tempo de $\mathrm{CEC}^{19}$.

Após a alta hospitalar, seis $(11,7 \%)$ pacientes foram readmitidos, todos por motivos relacionados ao procedimento cirúrgico. As principais causas foram infecção respiratória e ISC. Em um estudo descritivo, realizado no Sudeste brasileiro, os autores identificaram taxa de readmissão menor (5,9\%) do que a do presente estudo, sendo igualmente a ISC a principal causa. Entre os fatores que contribuem para ISC, estão DM, dislipidemia, obesidade, doença pulmonar obstrutiva crônica (DPOC) e tabagismo ${ }^{6}$. A mediastinite foi o foco de pesquisa de um estudo realizado na Universidade de São Paulo (USP), no qual a maior parte dos pacientes apresentou a infecção após a alta hospitalar (54,7\%) e, destes, 85,1\% necessitaram de reinternação, ficando hospitalizados, em média, 31,8 dias $^{20}$.

As complicações ocorridas no intraoperatório e no POI ocasionam maior tempo de internação, e os pacientes ficam vulneráveis a readmissões. O sangramento no intraoperatório, a ventilação mecânica prolongada, a dificuldade de estabilização da glicemia, entre outros, são fatores desencadeantes para um insucesso na alta hospitalar.

Dessa forma, as medidas de controle e prevenção são essenciais e estão diretamente relacionadas à evolução do paciente no pós-operatório. Programas de atenção à saúde que ofereçam cuidados pré-operatórios aos pacientes portadores de doenças preexistentes têm a finalidade de otimizar o tratamento, evitando a descompensação clínica no pós-operatório, com repercussões após a alta hospitalar ${ }^{6,21}$. Estudo realizado com 20 pacientes submetidos à cirurgia cardíaca reconstrutora mostrou que a maioria deles não foi orientada a respeito dos cuidados com a incisão cirúrgica, nem recebeu informações sobre sinais e sintomas de infecção no local da incisão. Evidencia-se, neste estudo, uma lacuna na transmissão das orientações, sendo estas realizadas somente no momento da alta hospitalar ${ }^{22}$. Uma reabilitação adequada, aliada aos cuidados domiciliares, é fundamental para que não ocorram reinternações. O controle e o cuidado com as comorbidades são fundamentais. Dessa forma, a atuação da equipe multidisciplinar é imprescíndivel para obtenção do sucesso no pós-operatório ${ }^{6}$.

A mortalidade neste estudo foi de três $(5,5 \%)$ pacientes. Dois deles apresentaram tempo de CEC prolongado (214 min e $136 \mathrm{~min}$ ), sendo esse um fator de risco para mortalidade. Os óbitos ocorreram nas primeiras 48 horas após o procedimento anestésico-cirúrgico; em duas situações, houve necessidade de reintervenção por sangramento, e, em outra situação, o paciente teve síndrome vasoplégica. A literatura nacional demonstra taxa de mortalidade variando de 8,7 a $14,2 \%{ }^{23,24}$. Quando associados à endocardite infecciosa, esses índices aumentam ainda mais, variando de 15 a $30 \%$, atingindo incidência de $10,5 \%$ em pacientes que desenvolveram mediastinite ${ }^{20}$.

As variáveis clínicas e sociodemográficas demonstram resultados compatíveis com outras pesquisas relacionadas à temática em questão. Reafirma-se, por este estudo, a importância em conhecer o perfil dos pacientes que realizam cirurgia cardíaca, com intuito de contribuir com orientações durante a internação, subsidiando estratégias para melhorar a adesão ao tratamento e ao controle das complicações.

Como limitação deste estudo, descreve-se o fato de a amostra ser pequena, já que se trata de um hospital com menor volume de intervenções cirúrgicas ao mês.

\section{CONCLUSÃO}

A maioria dos pacientes cujos dados foram analisados é do sexo masculino, com comorbidades, que realizaram procedimentos de trocas valvares e revascularização do miocárdio. A taxa de mortalidade foi de 5,5\%, e os óbitos ocorreram até 48 horas após o procedimento anestésico-cirúrgico. As complicações evidenciadas no período pós-operatório foram majoritariamente arritmias, sangramento e vasoplegia. A principal causa de readmissão, em 30 dias após a alta, ocorreu por infecção respiratória/derrame pleural e infecção de sítio cirúrgico.

O conhecimento do perfil e a evolução dos pacientes que realizaram a cirurgia cardíaca no hospital sede do estudo subsidiam estratégias para que melhorias nos processos assistenciais sejam implementadas, bem como ações de treinamento continuado para as equipes assistenciais, promovendo práticas seguras. Além disso, orientações para alta, voltadas à reabilitação cardíaca dos pacientes submetidos à cirurgia, devem ser ampliadas e diversificadas, incluindo os familiares e empoderando o paciente e a equipe de enfermagem perioperatória. 


\section{REFERÊNCIAS}

1. Brasil. Ministério da Saúde. Sistema de Informações de Mortalidade (SIM) e Instituto Brasileiro de Geografia e Estatística (IBGE) [Internet]. 2019 [acessado em 2 dez 2019]. Disponível em: http://www2.datasus. gov.br/DATASUS/index.php?area $=0203$

2. Contrin LM, Beccaria LM, Rodrigues AMS, Werneck AL, Castro GP, Teixeira CV. Complicações pós-operatórias cardiocrirúrgicas e tempo de internação. Rev Enferm UFPE. 2018;12(8):2105-12. https://doi. org/10.5205/1981-8963-v12i8a234846p2105-2112-2018

3. Heck LGS, Dallazen F, Cruz DT, Berwanger SA, Winkelmann ER. Análise do periodo intra e pós-operatório, complicações e mortalidade nas cirurgias de revascularização do miocárdio e de troca valvar. SCI Med [Internet]. 2017 [acessado em 23 mar. 2020]:27(4):ID28041. Disponível em: https://pesquisa.bvsalud.org/portal/resource/pt/biblio-876936

4. Dordetto PR, Pinto GC, Rosa TCSC. Pacientes submetidos à cirurgia cardíaca: caracterização sociodemográfica, perfil clínico-epidemiológico e complicações. Rev Fac Ciênc Méd Sorocaba. 2016;18(3):144-9. https://doi.org/10.5327/Z1984-4840201625868

5. Lima KCS, Moraes I, Lima AMF, Torres MT, Xavier CL, Gardenghi G. Qualidade de vida em idosos cardiopatas pré e pós-cirurgia cardíaca. RPF. 2017;7(2):171-8. http://doi.org/10.17267/2238-2704rpf.v7i2.1289

6. Barreiros BRN, Bianchi ERF, Turrini RNT, Poveda VB. Causas de readmissão hospitalar após cirurgia cardíaca. Rev Eletr Enf. 2016;18:1182. https://doi.org/10.5216/ree.v18.39529

7. Brasil. Ministério da Saúde. DATASUS. Procedimento Hospitalar do SUS: por local de internação: Brasil [Internet]. Brasília: Ministério da Saúde; 2019 [acessado em 2 dez. 2019]. Disponível em: http:// www2.datasus.gov.br/DATASUS/index.php?area=0202\&id $=11633$

8. Coppetti LC, Stumm EMF, Benetti ERR. Considerações de pacientes no pós-operatório de cirurgia cardíaca referentes às orientações recebidas do enfermeiro. REME. 2015;19(1):113-9. http://doi. org/10.5935/1415-2762.20150010

9. Silva CCF, Mello MB, Real AA, Albuquerque IM. Perfil clínico de pacientes submetidos à cirurgia de revascularização do miocárdio e troca valvar em um hospital terciário da região Sul do Brasil. Saúde (Santa Maria). 2019;45(2):1-11. https://doi.org/10.5902/2236583436298

10. Reis MMR, Lima EFA, Casagrande RI, Fioresi M, Leite FMC, Primo CC. Perfil epidemiológico de pacientes submetidos à cirurgia cardíaca. Rev Enferm UFPE. 2019;13(4):1015-22. https://doi. org/10.5205/1981-8963-v13i04a238020p1015-1022-2019

11. Vieira CAC, Soares AJC. Perfil clínico e epidemiológico dos pacientes que realizaram cirurgia cardíaca no hospital sul fluminense-HUSF. Rev Saúde. 2017;8(1):3-7. https://doi.org/10.21727/rs.v8i1.607

12. Li M, Zhang J, Gang TJ, Qin G, Wang L, Zhu M, et al. Enhanced recovery after surgery pathway for patients undergoing cardiac surgery: a randomized clinical trial. Eur J Cardiothorac Surg. 2018;54(3):4917. https://doi.org/10.1093/ejcts/ezy100

13. Silveira RC, Santos KMB, Moraes PAM, Souza NE. Desfechos clínicos de pacientes submetidos à cirurgia cardíaca em um hospital do noroeste do Rio Grande do Sul. REUFSM. 2016;6(1):102-11 https:// doi.org/10.5902/2179769216467
14. Torres PSS, Duarte TTP, Magro MCS, Lesão renal aguda: problema frequente no pós-operatório de cirurgia valvar. Rev Enferm UFPE. 2017;11(11):4311-8. https://doi.org/10.5205/ reuol.23542-49901-1-ED.1111201706

15. Dorneles CC, Bodanese LC, Guaragna JCVC, Macagnan FE, Coelho JC, Borges AP, et al. 0 impacto da hemotransfusão na morbimortalidade pós-operatória de cirurgias cardíacas. Rev Bras Cir Cardiovasc. 2011;26(2):222-9. https://doi.org/10.1590/S0102-76382011000200012

16. Sociedade Brasileira de Cardiologia. II Diretrizes Brasileiras de Fibrilação Atrial. Arq Bras Cardiol [Internet]. 2016 [acessado em 4 abr. 2020];106(4 Supl. 2):1 1-16. Disponível em: http://publicacoes.cardiol. br/2014/diretrizes/2016/02_II\%20DIRETRIZ_FIBRILACAO_ATRIAL.pdf

17. Ronsoni RM, Leiria TLL, Silvestrini TL, Martins LP, Krise ML, Silva RG, et al. Medidas profiláticas para fibrilação atrial no pós-operatório de cirurgia cardíaca. RELAMPA. 2018;31(2):38-44. https://doi. org/10.24207/1983-5558v31.2-001

18. Lopes ROP, Castro J, Nogueira CSC, Braga, DV, Gomes JR, Silva RC, et al. Complicações do pós-operatório imediato de cirurgia cardíaca eletiva: estudo transversal à luz de Roy. Rev Enf Ref. 2019;4(22):2332. http://doi.org/10.12707/RIV19042

19. Beccaria LM, Cesarino CB, Werneck AL, Correio NCG, Correio KSS, Correio MMM. Complicações pós-operatórias em pacientes submetidos à cirurgia cardíaca em hospital de ensino. Arq Ciênc Saúde [Internet]. 2015 [acessado em 20 maio 2020];22(3):37-41. Disponível em: http://www.cienciasdasaude.famerp.br/index.php/ racs/article/view/216/116

20. Kanasiro PS, Turrini RNT, Poveda VB. Perfil clínico-cirúrgico de pacientes com mediastinite pós-cirurgia cardíaca: estudo transversal retrospectivo. Rev SOBECC. 2019;24(3):139-45. http://doi.org/10.5327/ Z1414-4425201900030005

21. Braz NJ, Evangelista SS, Garbaccio JL, Oliveira AC. Infecção do sítio cirúrgico em pacientes submetidos a cirurgias cardíacas: uma análise de perfil epidemiológico. RECOM. 2018;8:1793. https://doi. org/10.19175/recom.v8i0.1793

22. Romanzini AE, Jesus APM, Carvalho E, Sasaki VDM, Damiano VB, Gomes JJ. Orientações de enfermagem aos pacientes sobre o autocuidado e os sinais e sintomas de infecção de sítio cirúrgico para a pós alta hospitalar de cirurgia cardíaca reconstrutora. REME [Internet]. 2010 [acessado em 20 maio 2020];14(2):239-43. Disponível em: https://cdn.publisher.gn1.link/reme.org.br/pdf/v14n2a14.pdf

23. Monteiro GM, Moreira DM. Mortalidade em cirurgias cardíacas em hospital terciário do sul do Brasil. Int J Cardiovasc Sci. 2015;28(3):2005. https://doi.org/10.5935/2359-4802.20150029

24. Santos CA, Oliveira MAB, Brandi ACB, Botelho PHH, Brandi JCM, Santos MA, et al. Risk factors for mortality of patients undergoing coronary artery by-pass graft surgery. Rev Bras Cir Cardiovasc. 2014;29(4):513-20. https://doi.org/10.5935/1678-9741.20140073

25. Guiomar N, Silva MV, Mbala D, Pinto BS, Monteiro JP, Ponce P, et al. Cirurgia cardíaca na endocardite infecciosa e preditores de mortalidade intra-hospitalar. Rev Port Cardiol. 2020;39(3):137-49. https://doi.org/10.1016/j.repc.2019.08.009 